\title{
A CHARACTERIZATION OF DISCRETE SOLVABLE MATRIX GROUPS
}

\author{
BY LOUIS AUSLANDER ${ }^{1}$
}

Communicated by N. Jacobson, January 17, 1961

Introduction. In [1], we introduced a class of solvable groups which we called algebraic strongly torsion free $S$ groups. We will show in this note how these groups can be modified and used to characterize those solvable groups which can be imbedded as discrete subgroups of the group $G L(n, C)$ for some $n$.

1. Preliminary discussion and definitions. Let $\Gamma$ be a strongly torsion free $S$ group in the sense of H. C. Wang [5]; i.e., $\Gamma$ satisfies the diagram

$$
1 \rightarrow D \rightarrow \Gamma \rightarrow Z^{S} \rightarrow 1
$$

where $D$ is a finitely generated torsion free nilpotent group and where $Z^{S}$ is the additive group of integers taken $s$ times. It is shown in [1] that there exists a unique maximal nilpotent subgroup $M$ of $\Gamma$ which contains the commutator subgroup $[\Gamma, \Gamma]$. Clearly $M$ is a characteristic subgroup of $\Gamma$ and torsion free. For any finitely generated torsion free nilpotent group $G$, we will use $N(G)$ to denote the unique connected simply connected nilpotent Lie group which contains $G$ as a discrete uniform subgroup. We will use $N_{C}(G)$ to denote the complexification of this Lie group. With this convention made, we may now let $A_{1}(\Gamma)$ denote the image of $\Gamma$ in the automorphism group of $N_{C}(M), A\left(N_{C}(M)\right)$, obtained by forming inner automorphisms of $\Gamma$. Let $\Gamma^{*} \subset \Gamma$ be a characteristic subroup of $\Gamma$ such that $\Gamma / \Gamma^{*}$ is finite, $\Gamma^{*} \supset M$ and $\Gamma^{*} / M$ is torsion free. We may apply the construction of H. C. Wang [5] to the group $S=\Gamma^{*} N_{C}(M)$ and obtain $S \subset F \cdot T$, where $F$ is the maximal unipotent subgroup, $F \supset N_{C}(M)$ as a characteristic subgroup, $T$ is abelian and the dot denotes semi-direct products. We may form $A_{1}(F) \subset A\left(N_{C}(M)\right)$.

Definition. We will say that a strongly torsion free $S$ group is complex algebraic if there exists an abelian analytic group of semisimple elements $T^{*}$ in $A\left(N_{C}(M)\right)$ such that

1. $T^{*}$ is in the normalizer of $A_{1}(F)$,

2. $A_{1}(\Gamma) \subset A_{1}(F) \cdot T^{*}$

where the dot denotes the semi-direct product.

REMARK 1. $T^{*}$ can be considered as an abelian analytic semisimple group of automorphisms of $N_{1}(F)$, where $N_{1}(F) \supset F, N_{1}(F)$ is connected simply connected nilpotent Lie group and $N_{1}(F) / F$ is compact.

${ }^{1}$ Research supported by N.S.F. Grant 15565 and O.O.R. contract SAR-DA-19020 ORD-5254. 
REMARK 2. $\Gamma^{*}$ is a discrete subgroup of $N_{1}(F) \cdot T^{*}$.

\section{Main theorem.}

THEOREM. A necessary and sufficient condition for a solvable group $\Gamma$ to have a faithful discrete matrix representation is that $\Gamma \supset \Gamma^{*}, \Gamma^{*} a$ complex strongly torsion free $S$ group such that

1. $\Gamma^{*}$ is normal in $\Gamma$ and $\Gamma / \Gamma^{*}$ is finite.

2. The group of automorphisms of $\Gamma^{*}$ induced by inner automorphisms of $\Gamma$ can be extended to $N_{1}(F) \cdot T^{*}$.

Proof of sufficiency. Consider the diagram

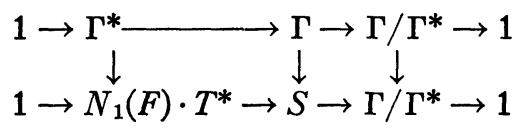

Then there exists one and only one group $S$ satisfying this diagram with the action induced from 2 above. By a theorem of Mostow [4], we have $S=E K$, where $E$ is a euclidean space and $K$ is a compact group. Hence the identity component $K_{0}$ of $K$ is abelian and $K=K_{0} \cdot \Gamma / \Gamma^{*}$ where the dot denotes the semi-direct product. Hence if $S_{0}$ is the identity component of $S, S_{0}=E K_{0}=N_{1}(F) \cdot T^{*}$ and $S=S_{0} \cdot \Gamma / \Gamma^{*}$. But $S_{0}=N_{1}(F) \cdot T^{*}$ has a faithful matrix representation by the Birkhoff theorem. Since $\Gamma / \Gamma^{*}$ is finite, this means that $S$ has a faithful matrix representation and hence so does $\Gamma$ contained in $S$.

ProOF of NECESSITY. Let $\Gamma$ be a discrete solvable subgroup of $G L(n, C)$. Let $H()$ denote the algebraic hull of the group in the bracket and let $H_{0}()$ denote the identity component of $H()$. Let $\Gamma_{1}=\Gamma \cap H_{0}(\Gamma)$. Then $\Gamma_{1}$ is a normal subgroup of $\Gamma$ and $\Gamma / \Gamma_{1}$ is finite. Further $H_{0}(\Gamma)$ is a solvable analytic group. Now let $\Gamma_{1}^{*}$ be a characteristic subgroup of $\Gamma$, of finite index all of whose eigen values are 1 or $\cos 2 \pi \zeta+i \sin 2 \pi \zeta$ where $\zeta$ is irrational. Clearly $\Gamma_{1}^{*}$ is normal in $\Gamma, \Gamma / \Gamma_{1}^{*}$ is finite and every automorphism of $\Gamma_{1}^{*}$ can be extended to $H\left(\Gamma_{1}^{*}\right)$. Further $\Gamma^{*}$ is clearly a strongly torsion free $S$ group. Let $\Gamma^{*}=\Gamma_{1}^{*} \cap H_{0}\left(\Gamma_{1}^{*}\right)$. Then it is trivial to verify that $\Gamma^{*}$ satisfies requirements. It is worth noting that one can actually prove that $\Gamma^{*}=\Gamma_{1}^{*}$. Since we do not need this result we will omit it.

\section{REFERENCES}

1. L. Auslander, Discrete uniform subgroups of solvable Lie groups, Trans. Amer. Math. Soc., to appear.

2. - Discrete solvable matrix groups, Proc. Amer. Math. Soc. vol. 11 (1960) pp. $687-688$.

3. A. Borel, Groupes linéares algébriques, Ann. of Math. vol. 64 (1956) pp. 20-82.

4. G. D. Mostow, Self-adjoint groups, Ann. of Math. vol. 62 (1955) pp. 44-55.

YALE UNIVERSITY and

INDIANA UNIVERSITY 